\title{
Zeitgemäßes über ein Virus und den Tod
}

\section{Einige klinische Beobachtungen}

\author{
Stephan Schmidt
}

Eingegangen: 6. November 2020 / Angenommen: 7. Dezember 2020 / Online publiziert: 26. Januar 2021

(C) Springer Fachmedien Wiesbaden GmbH, ein Teil von Springer Nature 2021

Zusammenfassung Der Artikel befasst sich mit der Frage, welchen Einfluss die aktuelle Covid-19-Pandemie auf tiefenpsychologische und analytische Therapie- und Supervisionsprozesse hat. Wie frei kann die „frei schwebende Aufmerksamkeit“ sein, wenn eine mächtige äußere Realität jede Begegnung begleitet? Die Pandemie konfrontiert uns zudem mit dem Tod und mit der psychisch in ihm repräsentierten Destruktivität und könnte Anlass sein, neu über die Theorie vom Todestrieb nachzudenken.

Schlüsselwörter Pandemie $\cdot$ Psychoanalytische Behandlungstechnik · Todestrieb

\section{Contemporary views about a virus and death}

Some clinical observations

\begin{abstract}
The article deals with the question, what influence the current Covid19 pandemic has on depth psychological and analytical therapy and supervision processes. How free can "evenly suspended attention" be when a powerful external reality accompanies every encounter? The pandemic confronts us with death and the destructiveness represented by its psychological aspects and could be an occasion to rethink the theory of the death instinct.
\end{abstract}

Keywords Pandemic Psychoanalytic treatment $\cdot$ Death instinct

Dr. med. S. Schmidt $(\triangle)$

Gartenstr. 15 a, 86911 Dießen, Deutschland

E-Mail: drstschmidt@aol.com 
Der Titel dieser Überlegungen bezieht sich auf eine Arbeit, die Freud 1915 unter dem Eindruck der ersten Monate des 1. Weltkrieges schrieb: „Zeitgemäßes über Krieg und Tod“ (Freud 1915 a). In dem ersten der zwei Essays („Die Enttäuschung des Krieges“) heißt es einleitend:

Von dem Wirbel dieser Kriegszeit gepackt, einseitig unterrichtet, ohne Distanz von den großen Veränderungen, die sich bereits vollzogen haben oder zu vollziehen beginnen, und ohne Witterung der sich gestaltenden Zukunft, werden wir selbst irre an der Bedeutung der Eindrücke, die sich uns aufdrängen, und an dem Werte der Urteile, die wir bilden. Es will uns scheinen, als hätte noch niemals ein Ereignis so viel kostbares Gemeingut der Menschheit zerstört, so viele der klarsten Intelligenzen verwirrt, so gründlich das Hohe erniedrigt. Selbst die Wissenschaft hat ihre leidenschaftslose Unparteilichkeit verloren ...

Ein Text, dessen Aktualität fast erschreckend ist: Ohne Witterung der sich gestaltenden Zukunft? Irrewerdend an der Bedeutung der Eindrücke? Zerstörung von kostbarem Gemeingut? Verwirrung der klarsten Intelligenzen? Selbst die Wissenschaft hat ihre Unparteilichkeit verloren? Nun handelt es sich derzeit unter dem Eindruck einer Virus-Pandemie zwar nicht um Krieg, aber doch um eine der unmittelbarsten und direktesten Bedrohungen, die die Nachkriegsgenerationen zu bewältigen haben. Vietnam-Krieg, Ebola, 11. September, auch Tschernobyl und Fukushima und viele Naturkatastrophen waren dann doch eher weit weg. Und bei dem HIV-Virus gab es zumindest die Idee, durch angemessen vorsichtiges Verhalten sich selber und Andere schützen zu können. Aber die Aerosole ... - auf die komme ich noch zurück.

Ich möchte zwei Aspekte kurz beleuchten: Was macht das Virus in unserer Praxis als analytische und tiefenpsychologische Therapeuten? Und: Wie gehen wir damit um, dass wir täglich in den Nachrichten über den durch das Virus verursachten Tod einer bestimmten Zahl von Menschen informiert werden?

\section{1 „Freischwebend“": Aufmerksamkeit und Aerosole}

Mein Eindruck in den letzten Monaten seit Beginn der Corona-Pandemie ist, dass in Therapien und Supervisionen ein Thema eine Präsenz bekommen hat, wie ich es in dieser Form und Intensität in den vielen Jahren meiner therapeutischen Arbeit nicht erlebt habe: die Angst. Sie hat sich in mehreren Formen gezeigt: In den Wochen der ,lock down“ genannten Kontaktbeschränkungen haben viele Therapeuten ihre Praxen entweder geschlossen oder sind auf Ersatzformen wie Telephon- oder Videositzungen ausgewichen. In den vielen Video-Sitzungen, die ich in diesen Wochen (und zum Teil bis heute) gemacht habe, hat sich mir der Eindruck aufgedrängt, dass hier eine neue Variante des Trends zu einer „kognitiven Wende“ im Kontext der Psychoanalyse entstanden ist: In den Video-Sitzungen (für mich noch deutlicher in Telephon-Sitzungen) haben wir die Kontaktmöglichkeiten auf den optischen Kontakt und auf die Worte einengen müssen; damit hat sich schon aus dem Kontaktmedium eine weitgehende Reduzierung auf das gesprochene Wort ergeben. Aber der analytische Dialog ist mehr ,,als ein Austausch von Worten“, es gibt daneben die ursprünglichste Form der Kommunikation zwischen Menschen, und die ist körperlich, 
die geht über körperliche Wahrnehmung von Anziehung und Abstoßung, die geht über den Geruch und die anderen Kanäle unmittelbarer sinnlicher Wahrnehmung das gilt nicht nur für den Säugling, der seine Mutter zunächst am Geruch erkennen kann, sondern das gilt auch im Erwachsenen-Dialog, in dem viel mehr ausgetauscht wird als Worte. Aber eben auch: Aerosole ...

Der Lockdown war nicht nur ein obrigkeitlich verordnetes und epidemiologisch als notwendig erachtetes ,,social distancing“, sondern er hat in meiner Wahrnehmung auch die Funktion einer Angstbewältigung: Wo wir uns nicht direkt begegnen, müssen wir auch keine Angst haben, uns zu infizieren. In der Logik des Unbewussten, das eben nicht logisch, sondern bildhaft-symbolisch und assoziativ organisiert ist, kann das heißen, dass es nicht nur um den möglichen Vorgang einer Virusübertragung geht, sondern vor allem auch um die Vermeidung einer emotionalen „Ansteckung“. Diese „Ansteckung“ geschieht in unserer therapeutischen Arbeit dauernd, z. B. in einer „enactment“ genannten (oft zunächst unbewussten) Übernahme von Impulsen, Bildern und Ängsten unserer Patienten. Ohne uns solcher „Ansteckung“ auszusetzen, können wir aber nicht tiefenpsychologisch oder analytisch arbeiten! Die unter dem Vorzeichen des social distancing wirksamen Abwehrmechanismen sind Affektisolierung und Rationalisierung - Psychotherapie kann zum „Austausch von Worten“ werden und damit einen zentralen Teil ihres Instrumentariums verlieren.

Das findet seine Fortsetzung nach dem „Lockdown“: Die Maske im Gesicht ist das Bild der Veränderung der Person (für die Nicht-Lateiner: ,persona“ ist im Lateinischen die Maske z. B. des Schauspielers, durch die hindurch der Sprechende zu hören ist - sonare heißt klingen). Durch die Papier- oder Textil-Maske hindurch sprechen wir miteinander und vergewissern uns selber und unser Gegenüber, dass wir nicht giftig und ansteckend sind. Und die Aufforderung zu Hygiene und Desinfektion suggeriert ebenfalls, dass hier potenziell toxische Subjekte miteinander zu tun haben, die vor (,Hände waschen!“) oder nach der Sitzung (Desinfektion von kontaminierten Flächen!) wieder saubere Bedingungen herzustellen haben.

Eine Kollegin beschreibt in der Supervision, wie sie nach der Stunde mit einem sehr angespannten Patienten, der seine Hände in die Kante der Stuhllehne verkrampft hatte, diese Lehne zu desinfizieren versucht, dabei die Rillen im Holz mit dem Desinfektionsmittel nur mühsam erreichen kann.

Wie können wir uns davon befreien, uns „dekontaminieren“, dass wir den Patienten (und uns selbst) als potenziell infektiös erleben müssen, wie können wir uns reinigen von der virologisch-hygienisch erforderlichen Reinigungsprozedur? Der Patient ist ein potenziell toxisches Gegenüber? Nein, unsere Aufgabe als analytische Therapeuten ist es immer und in jedem Moment, uns bereit zu halten für eine „Ansteckung“, für die oft zunächst unbewusste Teilhabe an der inneren Welt der Patienten. Wenn wir uns von der Angst vor Ansteckung infizieren lassen, berauben wir uns damit unseres wichtigsten Werkzeugs.

All das kann unsere Grundhaltung als tiefenpsychologische oder analytische Therapeuten beeinflussen: Wie sollen wir uns in den inneren Zustand einer gleichschwebenden Aufmerksamkeit versetzen, wenn wir neben und mitten in unserer frei schwebenden Aufmerksamkeit die ebenfalls stets frei im Raum schwebenden Aero- 
sole erwarten dürfen? (NB: die sind übrigens immer da, wenn Menschen miteinander reden, nur hat uns das bisher nie besonders gestört.)

Von Donald Winnicott wird Folgendes berichtet: „Während die British Psycho-Analytical Society 1943 in einer wissenschaftlichen Sitzung intensiv und leidenschaftlich über $\mathrm{Haß}$ und Aggression diskutiert, beginnt ein Luftangriff der Deutschen auf London. Die Teilnehmer sind derart vertieft in ihre eigene hitzige intellektuelle Schlacht, daß er (Winnicott) schließlich aufsteht und sagt: ,Ich möchte Sie nur darauf hinweisen, daß gerade ein Luftangriff stattfindet". Spricht's und setzt sich wieder. Und Margaret Little fährt in der Schilderung dieser Szene fort: ,Keiner nimmt Notiz von seiner Bemerkung und die Versammlung geht weiter, als ob nichts wäre'." (Auchter 2002, S. 22)

Es kann nicht darum gehen, die Realitäten einer Virus-Pandemie zu verleugnen und sich in einen abgegrenzten analytischen Binnenraum zurückzuziehen, sondern wir haben die Aufgabe, gerade in der Wahrnehmung der Realitäten den Spiel- und Phantasieraum als ,,intermediären Raum“(Winnicott 1971, S. 121 ff.) zu sichern, das freie Assoziieren (das ohnehin ja ein kaum erreichbares Idealbild ist) und die frei schwebende Aufmerksamkeit (für die dasselbe gilt) immer wieder neu zu erarbeiten. Vielleicht hilft es dabei, uns immer wieder den realen Aspekt dieser Angst vor Augen zu führen: die Real-Angst, die sich auf die Zukunft unseres Gemeinwesens richtet, auf die wirtschaftliche Entwicklung (auch unsere eigene ...), auf weiter steigende Infektions- und Todeszahlen und auf den weiteren Verlauf der Pandemie mit seinen unabsehbaren Folgen. Hier bräuchten wir eine rationale, von ,leidenschaftsloser Unparteilichkeit“ geprägte Sicht, die aber offensichtlich nicht einfach zu erreichen ist.

In den letzten Monaten hatte ich den Eindruck, dass wir in den ersten Minuten von Therapie- und Supervisionssitzungen zunächst über die Pandemie reden müssen, vielleicht ähnlich wie im Alltagsdialog, in dem wir uns zunächst über das Wetter austauschen. Vielleicht hat das die Funktion, uns in der gemeinsamen Wirklichkeit zu verorten, uns gegenseitig zu vergewissern, dass wir wissen und anerkennen, was draußen gerade los ist.

Wir brauchen diesen Blick auf die Realität, um in der Therapiestunde unsere Aufmerksamkeit auf neurotische Aspekte der Angst (beim Patienten wie bei uns selber) richten zu können. Auch für die Übertragungsangst gilt, dass der Blick auf die realen Bedrohungen uns als Abwehr vor der Wahrnehmung der Übertragung dienen kann. Wie bei allen anderen Realitäten, mit denen wir es in der Therapie zu tun haben, gilt also behandlungstechnisch auch hier die Maxime (Menningers „Dreieck der Einsicht“; Menninger und Holzmann 1977, S. 176f.), zwischen der Ebene der Realobjektbeziehung, der Ebene der Beziehung zu den internalisierten (frühen) Objekten und der Ebene der Übertragungsbeziehung zu oszillieren, d.h. wir versuchen, unsere Aufmerksamkeit jeweils neu (frei-schwebend) auf das gerade am wichtigsten Erscheinende auszurichten. Das ist unter dem Eindruck sehr mächtiger äußerer Realitäten nicht ganz einfach. 
Hinzu kommt ein weiterer Aspekt: Die tagtägliche Meldung von Todeszahlen macht deutlich, dass es in der aktuellen Angst-Pandemie (wie in jeder Angst) um die Auseinandersetzung mit dem Tod geht.

\section{Reden wir über den Tod!}

Der 2. Essay in der oben genannten Schrift von Freud hat den Titel „Unser Verhältnis zum Tode“. Auch hier einige Zeilen aus der Einleitung:

Das zweite Moment, von dem ich es ableite, daß wir uns so befremdet fühlen in dieser einst so schönen und trauten Welt, ist die Störung des bisher von uns festgehaltenen Verhältnisses zum Tode.

Dies Verhältnis war kein aufrichtiges. Wenn man uns anhörte, so waren wir natürlich bereit zu vertreten, daß der Tod der notwendige Ausgang alles Lebens sei, daß jeder von uns der Natur einen Tod schulde und vorbereitet sein müsse, die Schuld zu bezahlen, kurz, daß der Tod natürlich sei, unableugbar und unvermeidlich. In Wirklichkeit pflegten wir uns aber zu benehmen, als ob es anders wäre. Wir haben die unverkennbare Tendenz gezeigt, den Tod beiseite zu schieben, ihn aus dem Leben zu eliminieren. Wir haben versucht, ihn totzuschweigen. (Freud 1915 a)

Auch dieser Zeitdiagnose werden wir uns vorbehaltlos anschließen können. Aber die kollektive (und sicher auch immer wieder individuelle) Verleugnung des Todes wird durch die tägliche Konfrontation mit den tagtäglichen Nachrichten über den Tod in Frage gestellt: Pro Jahr sterben in Deutschland etwa 940.000 Menschen, das sind 2600 Menschen pro Tag. Ich habe den Begriff der „Übersterblichkeit“ erst lernen müssen: Er bezeichnet die Differenz zwischen der statistisch (auf Grund der Durchschnittswerte der vergangenen Jahre) erwartbaren Zahl der Toten pro Monat und der aktuell gemeldeten Zahl: Diese der Corona-Pandemie zugeschriebene Differenz lag in Deutschland am Beginn der Pandemie zwischen 4 und $11 \%$, im Juni bei 0\% und im Frühherbst 2020 wieder bei ca. 6\% (Vergleichszeitraum: 2016-2019). In dem statistischen Begriff der „Übersterblichkeit“ liegt schon das subtile Angebot zur Verleugnung: Wäre das Pandemie-bedingte „Über“ nicht so präsent, dann könnte man die Sterblichkeit insgesamt schon wieder vergessen? In den Therapien wirkt sich das tägliche Zählen von Corona-Toten aus.

Eine Patientin berichtet, dass ihre kleine Tochter die im Kindergarten vermittelte Angst formulierte, ein Besuch bei den Großeltern könne diese töten.

Leider hat das Kind, epidemiologisch betrachtet, ja sogar recht - diese Angst ist Real-Angst.

Aber an dieser Stelle müssen wir uns kurz schütteln und uns auf unsere Aufgabe als Analytiker und Tiefenpsychologen konzentrieren: Wenn wir es mit dem Unbewussten zu tun haben, dann dürfen wir auch die Spielregeln annehmen, die das Unbewusste prägen: „Die Vorgänge des Systems Ubw sind zeitlos, d.h. sie sind nicht zeitlich geordnet, werden durch die verlaufende Zeit nicht abgeändert, haben überhaupt keinen Bezug zur Zeit“" (Freud 1915b). Und weil das Unbewusste 
die Zeit nicht kennt, kennt es auch nicht die Begrenztheit der Zeit, kann es den Tod (als zeitliches Ende) nicht denken. „Im Grunde glaub(t) niemand an seinen eigenen Tod oder, was dasselbe ist: im Unbewußten ... (ist) jeder von uns von seiner Unsterblichkeit überzeugt" (Freud 1915a). Im Traum stürzen wir aus großer Höhe, und sterben nicht nur nicht daran, sondern können das dank wundersamer Auferstehung im nächsten Traum noch einmal erleben. Der Primärprozess kann unvereinbare Gegensätze gleichzeitig erleben; er folgt nicht der Kausalität und auch nicht der Chronologie, im Traum ist unsere Kindheit Gegenwart, und der Tod ist nur ein Bild oder ein Symptom wie jedes andere, das verstanden und ggfs. gedeutet werden will. D.h. es ist eine therapeutische Notwendigkeit, uns eben nicht mit dem notwendigerweise erwartbaren realen Tod zu beschäftigen, wenn wir uns im intermediären Raum der Assoziationen bewegen und darin die Freiheit haben wollen, uns mit dem Unbewussten zu beschäftigen jenseits der an Logik, Realitätsprüfung und Chronologie orientierten Regeln des Sekundärprozesses.

Haben wir im Umgang mit dem Tod diesem etwas entgegenzusetzen? Die psychische Repräsentanz des Todes ist die Angst: Eine basale Erfahrung des Kindes ist die Angst, aus dem Dunkel von Schlaf und Nacht nicht mehr zu erwachen, diese primäre Erfahrung ist eine seelische Realität (im pavor nocturnus immer wieder erlebt), und in diesem Erleben geht es um die grundlegende Frage, ob die sichernden frühen Objekte Garanten dafür sein können, dass diese im Dunkel der Nacht lauernde Todesgefahr überlebbar ist. Eine der wichtigsten Aufgaben der frühen Objekte ist, dieses Überleben symbolisch zu repräsentieren: Das abendliche Ritual, der Teddybär, das Bild vom Schutzengel und die elterliche Präsenz garantieren das Überleben und schaffen - im Idealfall - die Möglichkeit, ein solches Sicherheit gebendes ideales Objekt internalisieren zu können. Es ist unschwer erkennbar, dass wir in dieser primären Suche nach unzerstörbarer Objektsicherheit eine Quelle der Religionen finden können, in denen unsterbliche Götter und die heilige Mutter Gottes den (seelischen) Kosmos zusammenhalten sollen (vgl. Rohde-Dachser 2009).

Ich pflege in meinen Therapien auf Zitate aus der Bibel zu verzichten. Einen Text habe ich in den letzten Monaten aber immer wieder zitiert, er stammt aus dem Alten Testament:

Herr, lehre uns bedenken, dass wir sterben müssen, auf dass wir klug werden.

(Psalm 90, 12)

Ich habe diesen Text immer so verstanden, dass es in der hier benannten „Klugheit“" nicht darum geht, rechtzeitig eine Lebensversicherung abzuschließen o. ä.. Sondern es geht um eine sehr besondere Herausforderung an unsere Bereitschaft zur Wahrhaftigkeit, zur Aufmerksamkeit, zum Wissen um unsere Vergänglichkeit und Zerstörbarkeit.

Aber vielleicht gehört es auch zu dieser Klugheit, dass wir anerkennen, nicht nur Opfer eines (notwendigerweise irgendwann) erwartbaren Todes zu sein, den das Virus aktuell sehr deutlich vergegenwärtigt. Ein Blick auf die Geschichte (nicht nur auf die deutsche Geschichte) verweist uns auf die Realität, dass Tod und Destruktion eben nicht nur durch ein von außen kommendes Virus entstehen, sondern dass diese Destruktion einen Aspekt unserer menschlichen Potenzen darstellt - gerichtet gegen Menschen, gegen die Natur, gegen die Kultur. Eines der umstrittensten Elemente 
in Freuds Theorie ist der „Todestrieb“, also die Vorstellung von einer psychischen Energie, die den Lebens- und Selbsterhaltungstrieben (dem „Eros“") entgegengesetzt ist (als ,Thanatos“) und die - nach außen gewandt - als Destruktion sich darstellen kann. Unter dem Einfluss von Ich-Psychologie, Bindungstheorien und Selbstpsychologie ist dieses Theorem weitgehend dem Vergessen anheimgefallen (vgl. Lohmann 1983). Aber eine nicht primär auf psychische Wellness, Arbeits- und Genussfähigkeit (und auf die Vorgaben der Psychotherapie-Richtlinien der Kassenärztlichen Bundesvereinigung) ausgerichtete Psychoanalyse könnte als kritische Wissenschaft auf die allerorten lauernde Destruktivität hinweisen. Sie könnte damit eine politische Praxis werden.

Und wenn das Virus uns zwingt, in diesem Zusammenhang grundlegende Modelle nicht nur der psychoanalytischen Zunft neu zu denken: warum sollten wir nicht klüger werden können?

\section{Literatur}

Auchter, T. (2002). Winnicott - oder: Die Sehnsucht, wirklich lebendig zu werden. Luzifer - Amor. Zeitschrift zur Geschichte der Psychoanalyse, 15(30), 7-45.

Freud, S. (1915a). Zeitgemäßes über Krieg und Tod. GW X, 324-355, StA Bd. IX (S. 33-60). Frankfurt/M: Fischer.

Freud, S. (1915b). Das Unbewußte. GW X, 264-309, StA Bd. III (S. 119-173). Frankfurt/M.: Fischer.

Lohmann, H.-M. (1983). Wie harmlos dürfen Psychoanalytiker sein? Notizen zur verdrängten Thanatologie. In H.-M. Lohmann (Hrsg.), Das Unbehagen in der Psychoanalyse (S. 50-59). Frankfurt, Paris: Qumran.

Menninger, K.A., \& Holzmann, P.S. (1977). Theorie der psychoanalytischen Technik. Stuttgart: frommann-holzboog.

Rohde-Dachser, C. (2009). Todestrieb, Gottesvorstellungen und der Wunsch nach Unsterblichkeit. Eine psychoanalytische Studie. Psyche - Z Psychoanal, 63(9/10), 973-998.

Winnicott, D. W. (1971). Vom Spiel zur Kreativität. Stuttgart: Klett-Cotta.

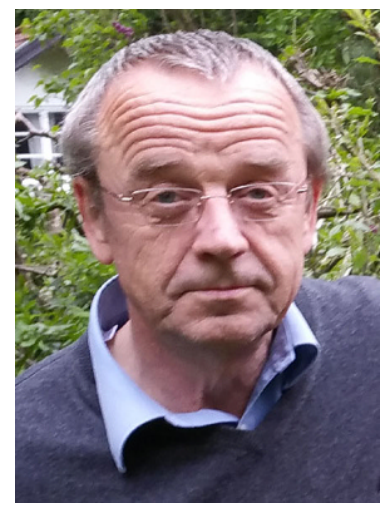

Dr. med. Stephan Schmidt studierte ev. Theologie und Humanmedizin, Facharzt für Psychiatrie und für Psychosomatische Medizin, seit 1992 Tätigkeit als Psychoanalytiker, Lehranalytiker und Supervisor in eigener Praxis, Dozent für Tiefenpsychologie und Psychoanalyse am ÄPK München, Konsiliararzt für Psychiatrie und für Psychosomatik in einer internistischen Klinik. Arbeitsschwerpunkte: Analytische Behandlungstechnik, psychotherapeutische Ethik. 